\title{
Comments on extracting the resonance strength parameter from yield data
}

\author{
Stephen Croft ${ }^{\mathrm{a}}$ and Andrea Favalli ${ }^{\mathrm{b}}$ \\ ${ }^{a}$ Safeguards \& Security Technology \\ Nuclear Security and Isotope Technology Division \\ One Bethel Valley Road, PO Box 2008, MS-6166 \\ Oak Ridge, TN 37831-6166, USA. \\ Phone: +18652412834 \\ e-mail: crofts@ornl.gov \\ ${ }^{\mathrm{b}}$ Safeguards Science \& Technology Group, \\ Non-proliferation and Nuclear Engineering Division \\ Los Alamos National Laboratory, MS E540, Los Alamos, NM 87545, USA. \\ Phone: +1 5056671429 \\ e-mail: afavalli@lanl.gov
}

The $F(\alpha, n)$ reaction is the focus of on-going research in part because it is an important source of neutrons in the nuclear fuel cycle which can be exploited to assay nuclear materials, especially uranium in the form of $\mathrm{UF}_{6}[1,2]$. At the present time there remains some considerable uncertainty (of the order of $\pm 20 \%)$ in the thick target integrated over angle $(\alpha, n)$ yield from ${ }^{19} \mathrm{~F}(100 \%$ natural abundance) and its compounds as discussed in [3] and [4]. An important thin target cross section measurement is that of Wrean and Kavanagh [5] who explore the region from below threshold ( $2.36 \mathrm{MeV}$ ) to approximately 3.1 $\mathrm{MeV}$ with fine energy resolution. Integration of their cross section data over the slowing down history of a stopping $\alpha$-particle allows the thick target yield to be calculated for incident energies up to $3.1 \mathrm{MeV}$. This trend can then be combined with data from other sources to obtain a thick target yield curve over the wider range of interest to the fuel cycle (roughly threshold to $10 \mathrm{MeV}$ to include all relevant $\alpha$ emitters). To estimate the thickness of the $\mathrm{CaF}_{2}$ target they used, Wrean and Kavanagh separately measured the integrated yield of the $6.129 \mathrm{MeV} \gamma$-rays from the resonance at $340.5 \mathrm{keV}$ (laboratory $\alpha$ particle kinetic energy) in the ${ }^{19} \mathrm{~F}(\mathrm{p}, \alpha \gamma)$ reaction. To interpret the data they adopted a resonance strength parameter of $(22.3 \pm 0.8) \mathrm{eV}$ based on a determination by Becker et al [6]. The value and its uncertainty directly affects the thickness estimate and the extracted $(\alpha, n)$ cross section values. In their citation to Becker et al's work, Wrean and Kavanagh comment that they did not make use of an alternative value of $(23.7 \pm 1.0) \mathrm{eV}$ reported by Croft [7] because they were unable to reproduce the value from the data given in that paper. The value they calculated for the resonance strength from the thick target yield given by Croft was $21.4 \mathrm{eV}$. 
The purpose of this communication is to revisit the paper by Croft published in this journal and specifically to explain the origin of the reported resonance strength. Fortunately the original notes spanning the period 12 January 1988 to 16 January 1990 were available to consult. In hindsight there is certainly a case of excessive brevity to rectify. In essence the step requiring explanation is how to compute the resonance strength, $\omega_{\gamma}$, from the reported thick target resonance yield $Y$.

For a single level isolated resonance the Breit-Wigner dispersion relationship describes the behavior of the $2(1,3) 4$ reaction cross section, $\sigma(E)$, as a function of the incident particle (particle 1 ) kinetic energy, $E$, in the laboratory frame. One way of writing this, which will prove convenient later, is as follows:

$\sigma(E)=\lambda^{2} \frac{(2 J+1)}{\left(2 S_{1}+1\right)\left(2 S_{2}+1\right)} \frac{\Gamma_{12} \Gamma_{34}}{\Gamma^{2}} \frac{\Gamma}{2} \frac{1}{\pi\left(1+\left(\frac{E-E_{R}}{\Gamma / 2}\right)^{2}\right)} \frac{1}{\left(\frac{\Gamma}{2}\right)}$

$\lambda$ is usually described as the de Broglie wavelength of the incident particle in the center of mass frame;

$E_{R}$ is the resonance energy, that is the value of the incident energy in the laboratory frame at which the cross section peaks;

The statistical weight factor, $g=\frac{(2 J+1)}{\left(2 S_{1}+1\right)\left(2 S_{2}+1\right)}$, expresses the various ways the angular momentum vectors can combine. $J$ is the angular momentum of the compound state, $S_{1}$ is the intrinsic angular momentum (spin) of the incident particle, $S_{2}$ is the intrinsic angular momentum (spin) of the target nucleus, and all three quantities are measured in units of $\hbar=h /(2 \pi)$, where $h$ is Planck's constant;

$\Gamma$ is the total reaction energy width of the resonance. It is the full width at half maximum value of the cross section. $\Gamma_{12}$ is the partial reaction energy width for re-emission of the incident particle, the formation channel. $\Gamma_{34}$ is the partial reaction width of the particular reaction 'decay' channel of interest. $\Gamma, \Gamma_{12}$ and $\Gamma_{34}$ all have units of energy (e.g. eV or keV) in the laboratory coordinate system;

With these definitions in place we see that the units of $\sigma(E)$ are those of area dependent on the choice of units for $\lambda^{2}$. The traditional nuclear physics unit is the barn where $1 \mathrm{~b}=10^{-24} \mathrm{~cm}^{2}$, with the understanding that it is the microscopic cross section per nucleus.

For the emergent channel of interest we define the reaction resonance strength parameter, $\omega_{34}$ as follows:

$\omega_{34}=g \frac{\Gamma_{12} \Gamma_{34}}{\Gamma}$ 
With this substitution the resonance integral for the decay channel of interest can be expressed as follows:

$$
A_{34}=\int_{-\infty}^{+\infty} \sigma(E) d E \approx \lambda_{R}^{2} g \frac{\Gamma_{12} \Gamma_{34}}{\Gamma^{2}} \frac{\Gamma}{2} \int_{-\infty}^{+\infty} \frac{1}{\pi\left(1+\left(\frac{E-E_{R}}{\Gamma / 2}\right)^{2}\right)} \frac{d E}{\left(\frac{\Gamma}{2}\right)}=\frac{1}{2} \lambda_{R}^{2} \omega_{34}
$$

where we have assumed that the resonance is narrow so that the explicit wavelength (incident particle energy) dependence, the energy dependence of the Coulomb barrier penetration factor, and any energy dependence of the resonance width parameters can all be ignored so that the cross section can be approximated by: $\sigma(E) \approx \sigma\left(E_{R}\right) /\left(1+\left(\frac{E-E_{R}}{\Gamma / 2}\right)^{2}\right)$. We have also made use of the well-known Cauchy probability distribution $\frac{1}{\pi\left(1+x^{2}\right)}$ which is normalized over the real number line, $x$. The limits of integration are not to be taken literally and it is sufficient for all practical purposes that $\left|\frac{E-E_{R}}{\Gamma / 2}\right| \gg 1$ at the extremities. Note the resonance integral (area), $A_{34}$, is independent of the reaction frame by conservation of probability.

The thick target resonance yield of a narrow resonance, that is the yield for an incident particle far above the resonance energy in units of $(\Gamma / 2)$ as it slows to far below the resonance energy in a target compound, is directly proportional to the resonance integral according to the following relationship which stems directly from the definition of the microscopic cross section:

$Y=w \frac{N_{A}}{A} \int \frac{\sigma(E)}{L(E)} d E \approx w \frac{N_{A}}{A} \frac{1}{L\left(E_{R}\right)} \int \sigma(E) d E=\left(w \frac{N_{A}}{A} \frac{1}{L_{R}}\right) A_{34}=w \frac{N_{A}}{A} \frac{1}{L_{R}} \frac{1}{2} \lambda_{R}^{2} \omega_{34}$

where $Y$ is the number of resonant reactions per incident particle, $w$ is the weight fraction of the target element in the target compound, $A$ is the molar mass of the target element, $N_{A}$ is Avogadro's number, and $L_{R}=L\left(E_{R}\right)$ is the mass stopping power of the incident ion in the target compound. For fluorine in the form of $\mathrm{CaF}_{2}$ the factor $(\mathrm{w} / \mathrm{A})$ can be replaced by $\left(2 / \mathrm{A}_{\mathrm{CaF}_{2}}\right)$ where $A_{\mathrm{CaF}_{2}}$ is the molar mass of $\mathrm{CaF}_{2}$. From the experiment summarized in [7] the value of $Y$ for the $6.129 \mathrm{MeV} \mathrm{Y}$-ray production from the $340.5 \mathrm{keV}$ (laboratory frame) resonance in the ${ }^{19} \mathrm{~F}(\mathrm{p}, \alpha \gamma)$ reaction is $1.397 \times 10^{-8}$ photons per proton with an estimated relative uncertainty at the one standard deviation level of $4.20 \%$. Note we are carrying additional significant figures solely to avoid rounding errors in later calculations. 
To estimate $\omega_{34}$ from $Y$ we need to find the numerical value of $\lambda_{R}^{2}$. For a target initially at rest in the laboratory frame and assuming non-relativistic kinematics we used in the original work:

$\lambda_{R}^{2}=\frac{(h c)^{2}}{2 \mu c^{2} E_{R}}=\frac{\left(\frac{h c}{m_{1} c^{2}}\right)^{2}}{2\left[E_{R} /\left(m_{1} c^{2}\right)\right] /\left(1+m_{1} / m_{2}\right)}=\frac{\lambda_{1}^{2}}{2\left[E_{R} /\left(m_{1} c^{2}\right)\right] /\left(1+m_{1} / m_{2}\right)}$

where $\mu=\frac{m_{1} m_{2}}{m_{1}+m_{2}}$ is the reduced mass of the pair of particles $m_{1}$ and $m_{2}, m_{1} c^{2}$ is the rest mass energy of the incident particle, and $\lambda_{1}=\frac{h c}{m_{1} c^{2}}$ is the Compton wavelength of the incident particle. With this expression, for protons on fluorine in $\mathrm{CaF}_{2}$ we obtain, by using the numerical values summarized in Table 1 , the resonance strength parameter value of $23.7 \mathrm{eV}$ as reported in [7]. This value is however wrong for two reasons. First the expression used to calculate $\lambda_{R}^{2}$ is incorrect if we are to follow the convention of using the center of mass frame to describe the parameters of the reaction. We observe that the single-level Breit-Wigner formula is discussed in many nuclear physics text books but often without clarity. The description by Evan's is salutary in this regard, however, where it is made clear that the incident particles are described as plane waves with a de Broglie wavelength of relative motion given by:

$\lambda=\frac{h}{\mu V_{r}}$

where $V_{r}$ is the mutual velocity of approach of the colliding particles whose reduced mass is $\mu$. Adopting non-relativistic transformations the relative speed of approach of the proton and the ${ }^{19} \mathrm{~F}$ target is readily shown to be $v_{1}$, the speed of the incident proton in the laboratory frame in which the ${ }^{19} \mathrm{~F}$ target is stationary, in both the laboratory and center of mass (center of momentum) frames. Thus we can write:

$\lambda_{R}^{2}=\frac{(h c)^{2}}{2 \mu c^{2} \frac{1}{2} \mu V_{r}^{2}}=\frac{\left(\frac{h c}{m_{1} c^{2}}\right)^{2}}{2\left[E_{R} /\left(m_{1} c^{2}\right)\right] /\left(1+m_{1} / m_{2}\right)^{2}}$

We see that the starting point superficially looks the same but that $\frac{1}{2} \mu V_{r}^{2}=\left(\frac{1}{2} m_{1} v_{1}^{2}\right) /\left(1+m_{1} / m_{2}\right)=$ $E_{1} /\left(1+m_{1} / m_{2}\right)$, the kinetic energy available in the center of mass frame, $E_{C M}$, evaluated at the resonance energy, $\left(E_{1}=E_{R}\right)$, replaces $E_{R}$ in the denominator. The difference in the final result is in the power of the factor $\left(1+m_{1} / m_{2}\right)$. The second reason is that $\omega_{34}$ has units of energy. It is not dimensionless. Its value therefore depends on the reference frame. We can see this more clearly by writing: 
$\omega_{34}=\left(g \frac{\Gamma_{12} \Gamma_{34}}{\Gamma^{2}}\right) \Gamma$

From which we see the resonance strength parameter is proportional to the total width. Just as $E_{C M}=E_{1} /\left(1+m_{1} / m_{2}\right)$ the total width parameters scales in the same way and so we can also write:

$\omega_{34, C M}=\frac{\omega_{34, L A B}}{\left(1+m_{1} / m_{2}\right)}$

The final expression for the resonance strength parameter in the center of mass frame, derived from the measured thick target resonance yield, becomes:

$\omega_{34, C M}=2 L_{R} \frac{1}{\left(w \frac{N_{A}}{A}\right)} \frac{2\left[E_{R} /\left(m_{1} c^{2}\right)\right] /\left(1+m_{1} / m_{2}\right)^{3}}{\lambda_{1}^{2}} Y$

This expression differs by a factor of $\left(1+m_{1} / m_{2}\right)$ squared compared to what was used originally used in [7]. We also observe that the value of the strength parameter calculated by Wrean and Kavanagh $(21.4 \mathrm{eV})$ and originally reported by Croft $(23.7 \mathrm{eV})$ differ numerically by this factor and so the discrepancy is understood and explained.

Based on the correct expression given above the following numerical relationship is obtained using the data given Table 1.

$\omega_{34, C M}=1.5308 \times 10^{9} \cdot Y\left(\gamma p^{-1}\right), e V=(21.38 \pm 0.99) \mathrm{eV}$

The uncertainty quoted was estimated by adding the three dominant fractional uncertainties in quadrature, these being, the $Y$ determination (4.20\%), the uncertainty in the resonance energy $(0.012 \%)$, and the estimated uncertainty in the mass stopping power (1.9\%) as discussed in [7]. We note that the corrected value is in excellent agreement with the value reported by Becker et al [6], (22.3 \pm 0.8$)$ $\mathrm{eV}$, obtained by averaging their two estimates, one based on normalization of the alpha particle yield to the Rutherford proton elastic scattering cross section and the other based on observation of the 
reaction gamma-rays, and a what we feel is a very well-conceived and executed experiment. It is not our purpose here to attempt a comprehensive evaluation of the strength parameter, but we note the weighted mean of [6] and [7] is $(21.94 \pm 0.62) \mathrm{eV}$, where the uncertainty is quoted as the internal standard deviation.

We note that although Wrean and Kavanagh decided to work through the strength parameter to obtain the thickness of their target, because they used $\mathrm{CaF}_{2}$, as did Croft, then the thick target resonance yield $Y\left(\gamma p^{-1}\right)$ could have been used directly and provides a natural and direct resonance strength standard without the additional mass stopping power uncertainty contribution.

Finally, we are grateful for this opportunity to set the record straight and hope our account helps others to avoid making similar mistakes. Our motivation is the reduction of the uncertainty associated with the nondestructive assay of $\mathrm{UF}_{6}$ for international nuclear safeguards and in the generation of quality evaluated nuclear data to support the first principles physics understanding of such applied measurements. Cross section data such as that reported in [5] are an important source of microscopic nuclear data. They used the $340 \mathrm{keV}{ }^{19} \mathrm{~F}(\mathrm{p}, \alpha \gamma)$ resonance strength parameter to determine the thickness if their targets. By improving the accuracy of the resonance strength parameter, as we have discussed here, we can therefore also reduce the derived cross section accuracy. In the future we also look forward to having available thick target neutron yield measurements for a range of fluorine compounds to a similar accuracy to those for some other for other light element targets, namely between 1-2 \% [10].

\begin{tabular}{|l|l|l|}
\hline Quantity & Value & Units \\
\hline Thick target resonance yield, $\mathrm{Y}$ & $1.39679 \times 10^{-8}$ & $6129 \mathrm{\gamma p}^{-1}$ \\
\hline $\begin{array}{l}\text { Resonance energy in the } \\
\text { laboratory frame, } E_{R}\end{array}$ & 340.46 & $\mathrm{keV}$ \\
\hline$\left(w \frac{N_{A}}{A}\right)=\left(2 \frac{N_{A}}{A_{C a F_{2}}}\right)$ & $1.54266 \times 10^{22}$ & $\mathrm{~g}^{-1}$ \\
\hline$\left(1+m_{1} / m_{2}\right)$ & 1.053047881 & \\
\hline Proton rest mass energy, $m_{1} c^{2}$ & 938272.31 & $\mathrm{keV}$ \\
\hline Proton Compton wavelength, $\lambda_{1}$ & $1.321410023 \times 10^{-13}$ & $\mathrm{~cm}^{-13}$ \\
\hline$L_{R}$ & 331750 & $\left(\mathrm{keVcm}^{-1}\right)\left(\mathrm{gcm}^{-3}\right)^{-1}$ \\
\hline
\end{tabular}

Table 1. Numerical data $[7,8]$ used for the evaluation of the resonance strength parameter based on the measurement reported in [7]. Note the yield is $8.718 \times 10^{4} \mathrm{\gamma} / \mu \mathrm{C}$ in the units used in [7] and using carrying an additional significant figure for the present work. The uncertainty in the resonance energy was taken to be $0.04 \mathrm{keV}$. 


\section{Acknowledgement}

This work was supported by the US DOE Office of Nonproliferation and Verification Research and Development, NA-22, as part of a project to improve uncertainty quantification and reporting. It also supports an effort to improve key nuclear data used in international nuclear safeguards, in particular a new measurement of the ${ }^{19} F(\alpha, n)$ cross section, which is how we became aware of the discrepancy identified by Wrean and Kavanagh.

\section{References}

[1] K. A. Miller, M. T. Swinhoe, S. Croft, T. Tamura, S. Aiuchi, and T. Iwamoto, Measured $F(\alpha, n)$ Yield from ${ }^{234} \mathrm{U}$ in Uranium Hexafluoride, Nuclear Science and Engineering 176(2014)98-105.

[2] E.B. Norman, T.E. Chupp, K.T. Lesko, P.J. Grant, and G.L. Woodruff, Addendum to " ${ }^{122}$ Na production cross sections from the ${ }^{19} \mathrm{~F}(\alpha, \mathrm{n})$ reaction", Phys. Rev. C 30, 1339 - Published 1 October 1984 arXiv1411.0051v2(2015) 10 January 2015.

[3] S. Croft, The thick target $(\alpha, n)$ yield of fluorine compounds for a broad range of actinides, 19th Annual ESARDA (European Safeguards Research and Development Association) Symposium on Safeguards and Nuclear Material Management, Montpellier, France, May 13-15, 1997. ESARDA 28 EUR 17665 EN(1997) 397-404.

[4] S. Croft, L C-A Bourva and C G Wilkins, The $(\alpha, n)$ Production Rate in Plutonium Fluoride, $25^{\text {th }}$ Annual Meeting ESARDA (European Safeguards Research and Development Association) Symposium on Safeguards and Nuclear Material Management, Stockholm, Sweden, 13-15 May 2003. EUR 20700 EN (2003) Paper P095. ISBN 92-894-5654-X.

[5] P.R. Wrean and R.W. Kavanagh, Total cross sections and reaction rates for ${ }^{19} \mathrm{~F}(\alpha, n)^{22} \mathrm{Na},{ }^{22} \mathrm{Ne}(\mathrm{p}, \mathrm{n})^{22} \mathrm{Na}$ and their inverses, Phys Rev C 62(2000)055805.

[6] H.W. Becker, W.E. Kieser, C. Rolfs, H.P. Trautvetter, and M. Wiescher, Resonance strengths of some light nuclei, Zeitschrift für Physik A 305(1982)319-323.

[7] S. Croft, The absolute yield, angular distribution and resonance widths of the $6.13,6.92$ and 7.12 $\mathrm{MeV}$ photons from the $340.5 \mathrm{keV}$ resonance of the ${ }^{19} \mathrm{~F}(p, \alpha \gamma){ }^{16} \mathrm{O}$ reaction, Nucl. Instrum. and Meths in Phys Res A307(1991)353-358.

[8] R.B. Firestone and V.S. Shirley (Eds), Table of Isotopes (Eighth Edition) Volume II: $A=151-272$ 
John Wiley \& Sons, Inc. (1996). ISBN 0-471-14918-7.

[9] R.D. Evans, The atomic nucleus,McGraw-Hill Book Company (1955).

[10] D. West and A.C. Sherwood. Measurement of thick target $(\alpha, n)$ yields from light elements, Ann. Nucl. Energy 9(1982)551-577. 